\section{METALLURGY}

\section{Artefact Analyses}

\section{from our Archaeology Correspondent}

THERE has been much effort recently toward the realization of a dream of prehistoric archaeologists-the determination, by the analysis of copper and bronze artefacts, of the source from which the constituent metal came. A successful solution to this problem would bring much useful information concerning early trade and the beginnings of metallurgy. But it now seems that the attainment of this goal is further away than ever, and that most of the data painstakingly accumulated may be of little value.

The underlying idea is that individual ore bodies are characterized by particular combinations of trace elements, so that analysis of early artefacts will repeat their characteristic "fingerprint". (Obviously this could work only for the early period, before the extensive remelting of used objects led to the mixing of copper from different sources.) There have been many analyses of the composition of the trace elements of different ore types from various localities. For example, following earlier analyses by Otto and Witter and by Neuninger and Pittioni, a Stuttgart group has divided thousands of objects, on the basis of the concentrations of various trace elements, into groups which supposedly reflect the place of origin of the metal (S. Junghans, E. Sangmeister and M. Schröder, Studien $z u$ den Anfangen der Metallurgie, 1, 1960 and 2, 1968).

The efficacy of the statistical method (H. Klein, Ber. Röm.-Germ. Komm., 34, 103 ; 1951) used by this team to group these analyses was always open to question because of its reliance on successive elements taken in turn. A much more persuasive average-link cluster analysis of the type now familiar in biological taxonomy has been carried out by F. R. Hodson (World Archaeol., 1, 90; 1969) who divided a hundred objects into sixteen groups on the basis of the Stuttgart analyses. Some individual groups have a wide geographical distribution, however, and a feature of the method is that all the elements considered are given equal weight.

J. A. Charles and E. A. Slater ( $A n$ tiquity, 44, 207; 1970) now question whether the Stuttgart analyses are repeatable to the level of accuracy used in their classificatory procedure. They show that segregation on cooling can cause very considerable variation in the concentrations of elements in different parts of the same object-up to 30 per cent in the case of bismuth. Because bismuth ranks prominently among the five elements used to effect the original classification into groups, the criticism is a damaging one. It reflects much less on Hodson's pro- cedure because this is a multivariate analysis, without yes/no decisions determined by the concentration of a single element.

Ultimately, however, the statistician and the metallurgist must face the problem presented by the ore bodies themselves. In how many cases can the ores of a given region be expected to yield metal which (1) regularly conforms to its supposed trace element "fingerprint", and (2) is not duplicated in composition by ores elsewhere? Clearly the first step must be a statistical consideration of different types of ores, of the kind begun by Friedman and his colleagues (Science, 152, 1504; 1966). Unfortunately it seems that the confidence levels for such statements may not be very high, and the archaeologist may be left to choose between the metallurgical "probability" that two objects found at opposite ends of Europe originated from the same ore and the archaeological "probability" that they did nothing of the sort.

The position does indeed look gloomy. The director of the Research Laboratory for Archaeology at the University of Oxford expressed a pessimistic view at a recent symposium (E. T. Hall in The Impact of the Natural Sciences on Archaeology (edit. by T. E. Allibone), Oxford University Press, 1970): "Our experience at Oxford has tended to show that such analyses are a waste of time. ... It is technological improvements in manufacturing technique which can be demonstrated by analysis of the major constituents of bronzes rather than the solution of attribution problems by trace analysis". Unfortunately, it seems that Hall may be right.

\title{
Galactic Helium from Massive Stars
}

FOR some time it has been believed that there is a maximum mass of about one hundred solar masses for stars. Above that mass, theoretical calculations predict that the stars are unstable and that the instability occurs rapidly compared with the evolution of the star. Recently, nonlinear calculations by several authors have suggested that the instability is significantly stabilized at large amplitudes and that the stars only lose mass at a rate comparable with that at which hydrogen is converted into helium by nuclear reactions inside them. If this is so, a considerable amount of helium could be produced in such stars and be expelled.

Talbot and Arnett suggest in next Monday's Nature Physical Science that this could be an important source of helium in our galaxy, although they admit that their estimates of helium production are rather crude at present. If this is so, there must have been a first generation of very massive stars when our galaxy was formed and detailed calculations would need to show that the stars have lost most of their mass after helium has been produced but before substantial production of heavy elements, which seem to be about ten times less abundant by mass than helium in young stars.

There is particular interest in the subject of helium in the universe because helium is the only element which is comparable in abundance with hydrogen in the part of the universe which we can observe directly. Recently it has been popular to suppose that the originally created matter consisted only of light elements and that the heavier elements have been built up by nuclear fusion reactions, probably inside stars. The crucial question is whether the matter which was originally created was hydrogen alone or a mixture of hydrogen and helium.

A few years ago it was found that all objects, for which reasonably reliable helium abundances existed, possessed at least 25 per cent of helium by mass. It was then realized that the hot big bang cosmological theory propounded by George Gamow predicted that nuclear reactions should have produced a mixture of hydrogen and helium after the first few minutes of the expansion of the universe, which could be regarded as the initial chemical composition of the universe. At that stage it was impossible to predict a precise amount of helium, but the discovery of the microwave background radiation changed the situation (A. E. Penzias and R. W. Wilson, Astrophys. J., 142, 419; 1965). The hot big bang theory requires the universe to be filled today with a blackbody distribution of microwaves and the temperature of the radiation determines the initial helium production. The temperature of about $2.7 \mathrm{~K}$ predicted a helium content close to 25 per cent (R. V. Wagoner et al., Astrophys. J., 148, 3; 1967).

Although this theory of primaeval helium production is very attractive, it cannot be valid if objects are discovered with little or no helium or if the microwave radiation proves not to be of blackbody form when all the relevant wavelengths have been studied. There have recently been observations which suggest both of these results. Although the primaeval origin of most of the helium has certainly not yet been disproved, it seems desirable to consider other possible origins of helium and this is what Talbot and Arnett have now done. 